ISSN: 1130-3743 - ISSN electrónico: 2386-5660

DOI: http://dx.doi.org/10.14201/teoredu2015272185201

\title{
EDUCAR PARA UM ENVELHECIMENTO BEM SUCEDIDO: REFLEXÕES E PROPOSTAS DE AÇÃO
}

\author{
Educar para un envejecimiento exitoso: reflexiones \\ y propuestas de acción
}

\section{Educate for successful aging: reflections and proposals for action}

\author{
Maria Conceição ANTUNES \\ Instituto de Educação. Universidade do Minho. Campus de Gualtar, 4710-057 \\ Braga.Portugal.mantunes@ie.uminho.pt
}

Fecha de recepción: marzo de 2015

Fecha de aceptación: junio de 2015

Biblid [(1130-3743) 27, 2-2015, 185-201]

RESUMO

O envelhecimento da população é, sem dúvida, um dos fenómenos mais preocupantes da atualidade, colocando sérios problemas e novos desafios às sociedades contemporâneas.

Neste ensaio, pretendemos convidar o leitor a refletir connosco acerca de alguns dos desafios que o envelhecimento da população coloca à vivência intracomunitária dos nossos dias, de um modo particular, os de natureza educacional.

A intenção é, em primeiro lugar, tentar desocultar as articulações que se estabelecem entre os conceitos de envelhecimento bem sucedido, qualidade de vida e saúde no sentido de compreender se o envelhecimento bem sucedido é uma realidade possível. Em segundo lugar, a nossa reflexão projeta uma incontornável relação entre educação e envelhecimento bem sucedido, tecendo algumas propostas de reconfiguração da educação de forma a esta poder ser entendida como fator promotor do envelhecimento bem sucedido e qualidade de vida na população idosa. 
Palabras chave: envelhecimento bem sucedido; qualidade de vida; saúde; educação.

\section{RESUMEN}

El envejecimiento de la población es, sin duda, uno de los fenómenos más inquietantes de nuestro tiempo, el cual da lugar a serios problemas y a nuevos desafíos para las sociedades contemporáneas.

En este ensayo nos proponemos invitar al lector a reflexionar sobre algunos de los retos más apremiantes que el envejecimiento de la población plantea al transcurrir del día a día en nuestras comunidades y, de manera particular, en el ámbito educativo.

La intención es, en primer lugar, tratar de descubrir las conexiones que se establecen entre los conceptos de envejecimiento exitoso, calidad de vida y salud, a fin de comprender si envejecer con éxito es una realidad posible. En segundo lugar, nuestra reflexión revela una relación inevitable entre la educación y el envejecimiento exitoso y articula algunas propuestas para reconfigurar la educación de forma tal que pueda ser entendida como un factor capaz de promover el envejecimiento exitoso y la calidad de vida entre la población de edad avanzada.

Palabras clave: envejecimiento exitoso; calidad de vida; salud; educación.

\section{SUMMARY}

The aging population is undoubtedly one of the most disturbing phenomenon of our time, posing serious problems and new challenges to contemporary societies.

In this essay, we intend to invite the reader to reflect with us about some of the compelling challenges that the aging population places to the intra-community experience of our days, in a particular way, those of education.

The intention is, first of all, try to uncover the joints that are established between the concepts of successful aging, quality of life and health in order to understand if successful aging is a possible reality. Secondly, our designs reflect an inescapable relationship between education and successful aging, weaving some proposals of reconfiguration of education so that this can be understood as a promoting factor of successful aging and quality of life of the elderly population.

Key words: successful aging; quality of life; health; education.

\section{INTRODUÇÃO}

A construção social e política do envelhecimento da população é, sem dúvida, um dos fenómenos mais preocupantes da atualidade, colocando sérios problemas e novos desafios às sociedades contemporâneas. 
Este, contudo, não é um fenómeno universal mas um problema localizado, inerente às sociedades ditas desenvolvidas. Ao analisarmos o envelhecimento das sociedades, através do desequilíbrio das respetivas estruturas etárias, constatamos o facto de ele atingir apenas algumas das sociedades do conjunto mundial, concretamente e de maneira sumária identificadas com as que têm beneficiado de posições dominantes na arena das relações mundiais (Esteves, 1995).

Efetivamente, as estruturas etárias dos Estados Unidos da América e da União Europeia denunciam uma realidade conhecida por envelhecimento demográfico, consubstancializado num aumento da esperança de vida à nascença que se traduz no aumento do grupo de pessoas idosas e numa diminuição da taxa de natalidade. A população, nestas sociedades, caracteriza-se por um duplo envelhecimento, um aumento do número de idosos e uma diminuição do número de jovens, dando origem à chamada transição demográfica caracterizada pela passagem de um modelo demográfico de fecundidade e de mortalidade elevados para um modelo em que ambos os fenómenos atingem níveis baixos (Rafart, 2001).

Uma vez conseguido o significativo aumento da esperança de vida, o grande desafio que se coloca às sociedades contemporâneas será fazer corresponder qualidade de vida a este aumento de anos. O desafio consiste não apenas em "dar mais anos à vida" mas "mais vida aos anos", o que pressupõe repensar o processo de envelhecimento e sistematizar novas formas de viver a longevidade, preservando os princípios da dignidade, da autonomia e da solidariedade através da criação de condições que permitam potenciar a qualidade de vida e bem-estar, proporcionando um aumento de anos de vida com qualidade (Fontaine, 2000; Osório e Cabral, 2007).

Envelhecer é, por si, um processo natural que se caracteriza por um conjunto de processos ou fenómenos que o organismo vai sofrendo com o avançar da idade provocando modificações biológicas e psicológicas que têm, consequentemente, repercussões sociais (Carvalho, 2012; Portella, 2004; Parente, 2006).

Não obstante o envelhecimento ser um processo natural que devamos aceitar com naturalidade, dado a velhice ser mais uma etapa da vida (Geis, 2003), esta deve ser preparada para que a possamos viver da melhor maneira possível. Da perspetiva em que nos colocamos face à questão do envelhecimento entendemos que se envelhecer é um processo natural, envelhecer com qualidade de vida é uma questão de educação. Podemos ir aprendendo ao longo da vida a envelhecer com qualidade em contacto com diferentes agentes educativos, quer em contextos educativos formais, quer em contextos educativos não formais e informais apostados em promover a educação na perspetiva de um processo de transformação individual na tripla dimensão do saber (conhecimentos), do saber fazer (habilidades) e do saber ser (atitudes) que permitam e promovam a qualidade de vida na última fase da vida.

Orientados por este horizonte de compreensão, entendemos que educação, envelhecimento bem sucedido, qualidade de vida e saúde são conceitos indissociáveis, porque profundamente interligados. 
Num primeiro momento da nossa reflexão, procuraremos explicitar a relação entre envelhecimento bem sucedido e qualidade de vida. Envelhecimento bem sucedido significa envelhecer com qualidade de vida? Mas que significa qualidade de vida? Qual a sua relação com a saúde entendida como bem-estar físico, mental, social e espiritual?

O segundo momento, invocando as questões anteriores e as cumplicidades e interações que se estabelecem entre os conceitos que as animam, procura compreender a relação entre educação e envelhecimento bem sucedido. Qual a importância e significado de educar para o envelhecimento bem sucedido nos nossos dias? Esta questão abre caminho à proposta de uma recontextualização da educação, no sentido de invocar o papel educativo no processo do envelhecimento bem sucedido configurando-o uma realidade possível.

\section{ENVELHECIMENTO BEM SUCEDIDO, QUALIDADE DE VIDA E SAÚDE}

O envelhecimento é um processo normal e dinâmico que embora se manifeste e evidencie de forma mais notória no fim da vida, tem início no momento do nascimento desenvolvendo-se ao longo da vida. Pode ser apreendido a diversos níveis: biológico dado que as características da velhice se traduzem com a idade nas modificações do nosso aspeto e num aumento das doenças; social pela mudança de estatuto provocada com a passagem à reforma e psicológico nas modificações das nossas atividades intelectuais, motivações e vínculos relacionais (Fontaine, 2000; Carvalho, 2012). Trata-se, segundo Atchley (2000, 526) de «um conjunto de processos de natureza física, psicológica e social que, com o tempo, produzem mudanças na capacidade de funcionamento dos indivíduos e influenciam a sua definição social" caracterizando-se pelo facto de ser universal, afetando, assim, todos os indivíduos.

Embora universal, este processo não é igual, nem acontece de forma idêntica em todas as pessoas, é um fenómeno influenciado por fatores genéticos, ambientais e fatores de risco vividos pelo indivíduo (Pérez, 2000) traduzindo-se num processo singular correlativo e consequente das dimensões fisiológica, psicológica e social de cada indivíduo.

Pressupondo que o envelhecimento bem sucedido corresponde à manutenção da qualidade de vida na fase mais avançada da vida e que a qualidade de vida se caracteriza por um sentimento de bem-estar, por um bom estado de saúde física, uma emotividade equilibrada e uma boa integração social, fará sentido falar de qualidade de vida na terceira idade? Qual a relação entre os conceitos de envelhecimento bem sucedido, qualidade de vida e saúde?

Em boa verdade, o envelhecimento bem sucedido é um constructo relativamente ao qual «não há acordo entre os autores, mas que, no entanto permite algum consenso no sentido de se relacionar com um mais elevado nível de funcionamento nos domínios físico, psicológico e social, implícitos numa boa adaptação» (Paúl, 1996, 20). É um facto que, nos nossos dias, as descobertas farmacológicas aliadas 
ao melhoramento das condições de vida e à elevação do nível cultural invocaram um aumento explosivo de pessoas idosas com bom funcionamento, boa saúde e com elevado nível de satisfação (Fontaine, 2000).

Falamos, então, de envelhecimento bem ou mal sucedido partindo da análise de padrões de adaptação do idoso às suas atuais capacidades de funcionamento, ou seja, do grau de satisfação e bem-estar que o idoso revela e perceciona, quer ao nível da sua vida pessoal, quer ao nível da sua vida social. Neste sentido, o envelhecimento bem sucedido pressupõe um baixo risco de doenças ou incapacidades relacionadas com a doença, um funcionamento físico e mental elevado e um empenhamento ativo na vida, ou seja, um relativamente elevado nível de saúde física, bem-estar psicológico e competência na adaptação (Wong, 1989).

No final da década de 90, dado o reconhecimento de que, para além da saúde, outros fatores afetam o modo como os indivíduos e as populações envelhecem, a Organização Mundial de Saúde procurando uma designação mais abrangente substituiu a designação "envelhecimento saudável» pela designação «envelhecimento ativo" definindo-o como «o processo de optimização das oportunidades de saúde, participação e segurança, no sentido de aumentar a qualidade de vida durante o envelhecimento" (OMS, 2002, 12). O envelhecimento ativo, enquanto processo de envelhecimento bem sucedido pressupõe como principal meta, melhorar a qualidade de vida das pessoas e aumentar a expectativa de uma vida saudável, contribuindo para a sua autonomia e independência, providenciando a satisfação das necessidades básicas de bem-estar físico e de segurança pessoal através de um ambiente que proporcione o desenvolvimento de laços sociais, e estabilidade emocional.

Neste horizonte de compreensão do processo de envelhecimento, envelhecer com sucesso é uma qualidade daqueles que se confrontam e se adaptam bem às mudanças que naturalmente acompanham o aumento progressivo de anos, promovendo estratégias positivas de recontextualização de formas de estar na vida mais adequadas às capacidades, recursos e motivações característicos de uma idade mais avançada de modo a manter o bem-estar. Neste sentido, o conceito de envelhecimento bem sucedido está relacionado com o conceito de bem-estar.

De modo genérico, o bem-estar é um conceito ambíguo e subjetivo, na medida em que integra dimensões muito heterogéneas que vão desde a satisfação de necessidades básicas, ao estado de saúde e ao nível de vida socioeconómico e cultural do indivíduo. Neste sentido, o bem-estar deve ser entendido como a satisfação das necessidades fundamentais do homem e dado o seu sentido totalizador, a expressão que melhor o designa seja a de qualidade de vida.

Com uma significação, de igual modo, abrangente e complexa, variando conforme as épocas e os padrões culturais, o conceito de qualidade de vida está relacionado com a forma como cada indivíduo perceciona e se relaciona com o mundo. A Organização Mundial de Saúde define-o como a "perceção dos indivíduos sobre a sua posição na vida, no contexto da cultura e do sistema de valores nos quais vivem e em relação aos seus objetivos, expectativas, preocupações e 
padrões de bem-estar» (OMS, 2002,13). Parece ser consensual a ideia de que o conceito qualidade de vida se refere ao modo como as pessoas vivem, sentem e compreendem o seu quotidiano (Pontes, 2012; Jacob, 2007). Significa isto que o conceito de qualidade de vida refere bem-estar físico (comodidade em termos materiais, saúde, higiene e segurança), bem-estar psicológico (relações interpessoais satisfatórias) e bem-estar social (participação na vida familiar e comunitária) (Donald, 1997).

A análise dos conceitos permite-nos afirmar que o envelhecimento bem sucedido parece estar, efetivamente, relacionado com qualidade de vida. Passaremos, então, a evidenciar a relação existente entre qualidade de vida e saúde.

Parece relevante fazermos referência ao facto de, não raramente, fazermos uma certa identificação entre o estado de saúde e qualidade de vida, considerando-se esta uma consequência direta do estado de saúde. No entanto, não obstante o facto de a saúde constituir um aspeto essencial na qualidade de vida do idoso, o peso que exerce continua a ser muito controverso pois, tanto quanto a saúde, o que parece influenciar determinantemente a qualidade de vida dos idosos são as vivências de perda de pertença, a falta de expectativas face ao futuro, a solidão, o sentido de inutilidade e as frustrações afetivas. Como Ramos (2001, 228) faz notar,

quando se investiga o modo como as pessoas avaliam a sua própria situação, em termos de aspectos positivos e negativos da sua vida quotidiana, verifica-se que, não obstante a saúde ser um factor importante, o que mais conta são as relações com os outros, o modo como usam o seu tempo e as emoções positivas que podem levá-las a aceitar os lados negativos da sua saúde e a suportar os eventuais efeitos molestos dos tratamentos.

O conceito "qualidade de vida" começou a vincular-se ao conceito de saúde quando a Organização Mundial de Saúde na tentativa de unificar critérios face à pluralidade de definições surgidas no pós-guerra define a saúde como «um estado de completo bem-estar físico, mental e social e não somente como ausência de doença ou enfermidade» (oms, 1948; 1978, 2), alertando para a importância da promoção da saúde não só ao nível do controlo de sintomas e no aumento da esperança média de vida mas, também, ao nível da promoção do bem-estar e da qualidade de vida das pessoas (Canavarro, 2010).

Esta definição reflete uma nova abordagem antropológica e uma viragem de paradigma relativamente ao tradicional modelo biomédico, sublinhando uma visão sistémica e holística do ser humano ao entender a saúde como resultado de múltiplas dimensões, física, mental e social -às quais se vem, mais tarde, a acrescentar a dimensão espiritual- explicitando a saúde de uma forma positiva, identificando-a com bem-estar.

Provavelmente o mais significativo desta definição tenha sido a introdução do conceito de bem-estar. [...] Um conceito que atuou como alavanca de ruptura dos tranquilos limites de uma medicina (ocidental) alojada no paradigma de 
saúde-enfermidade-morte e que abriu as portas a uma nova perceção da relação ser humano-ambiente bastante distinta da que reduzia as ameaças para a saúde aos micróbios, germes e vírus (Martínez, Sáez e Escarbajal, 2000, 21-29).

A adoção desta definição mais compreensiva, assente no modelo biopsicossocial que legitima uma definição de saúde multidimensional e exige uma prestação de amplos serviços (sociais, saúde, educação), uma avaliação da qualidade de vida dos pacientes e a sua duração (Kaplan, Sallis e Patterson, 1998), foi determinante na definição do constructo qualidade de vida (Levi e Drotar, 1998). Este conceito integra as dimensões centrais da definição de saúde, sendo mesmo expandidas no sentido de avaliar o estado de saúde e sintomas físicos, o estado funcional, a adaptação e funcionamento psicológico e social (Aaronson, 1988).

Tendo em consideração a definição da OMS (2002), que entende a qualidade de vida como a perceção do indivíduo face à sua própria vida, a qualidade de vida evidencia-se como um conceito amplo e subjetivo que inclui a saúde física da pessoa, o seu estado psicológico, o nível de independência, as relações sociais e as crenças e convicções pessoais. Esta noção inclui, assim, aspetos objetivos e comensuráveis como condição económica para viver uma vida condigna e aspetos subjetivos e incomensuráveis como a perceção da saúde física e mental e sentimentos de satisfação, autorrealização pessoal e felicidade.

Falar de qualidade de vida na terceira idade supõe, então, assegurar não apenas a satisfação das necessidades biológicas e bem-estar físico, mas também um ambiente de segurança pessoal, um meio social que favoreça o relacionamento interpessoal e o desenvolvimento cultural promotores da estabilidade psicológica e integração social. Entendida nesta perspetiva holista, a qualidade de vida constitui uma condição decisiva para o desenvolvimento humano, um estado positivo que compreende o indivíduo no seu todo e no contexto do seu estilo de vida (OMS, 1986; OMs, 2001; Pais Ribeiro, 2005).

Neste sentido, o conceito qualidade de vida é indissociável do conceito de saúde dado ambos, partindo de uma perspetiva holista, se relacionarem com o bem-estar físico, psicológico, afetivo e emocional, social, cultural e espiritual do ser humano, abarcando desde o bem-estar material, a não dependência e ausência de patologias limitadoras da capacidade funcional, até à perceção do desempenho nas diversas áreas da vida e respetiva satisfação pessoal global.

Não obstante o significativo salto qualitativo que representa na forma de se entender a saúde, esta definição tem sofrido numerosas críticas que denunciam, fundamentalmente, a dimensão utópica da expressão "completo bem-estar». Se bem que o completo bem-estar em todas as dimensões da vida seja difícil de alcançar, é um facto que a noção de saúde tem vindo a ser entendida no sentido cada vez mais integrador de uma multiplicidade de dimensões cujo equilíbrio dinâmico e complexo se identifica com bem-estar (Jenney, 1997; Ribeiro, 1994; Ribeiro, Meneses, Meneses e Grupo-QvD, 1998; Spieth e Harris, 1996).

Convocando Ángeles Murga (2004), diremos que a partir da Carta de Ottawa (1986) temos vindo a assistir à consolidação de um movimento internacional (New 
Public Health) que defende um conceito de saúde multidimensional e holístico, cuja ressignificação se evidencia em dois aspectos fundamentais: a) numa visão positiva enquanto um estado de bem-estar produto do equilíbrio integrado de todas as dimensões humanas-biológica, psicológica, social, espiritual, cultural e ambiental; b) numa visão holística e integral cuja responsabilidade é extensiva a todos os seres humanos, comunidades e contextos naturais.

O avanço das ciências da saúde e da vida permitiu evidenciar que, viver mais e bons anos é uma tarefa que depende de cada um de nós, enquanto agentes ativos e construtores/decisores da nossa história de vida, optando por estilos de vida saudáveis, no sentido de prevenir a doença, aliviar os sintomas provocados pelos desequilíbrios físicos e psíquicos, melhorar o funcionamento do organismo, fomentar as relações sociais e a autonomia e satisfação pessoal. A partir da Carta de Ottawa, e portanto durante as últimas décadas, os documentos internacionais têm vindo a apresentar recomendações e orientações para os sistemas nacionais de saúde procurando transmitir esta forma de entender a saúde, sensibilizando para o facto de a saúde e bem-estar não ser um produto ou responsabilidade exclusiva dos profissionais de saúde, mas sim uma questão a ser trabalhada do ponto de vista individual e comunitário. No caso específico de Portugal as mais recentes diretivas no âmbito da saúde presentes no Plano Nacional de Saúde (2011-2016) reafirmam claramente esta posição, responsabilizando os indivíduos enquanto cidadãos de direitos, deveres e poder de decisão, valorizando a sua capacitação para promoverem a sua saúde (ACS, 2011).

No seguimento das reflexões que fomos expondo, envelhecimento bem sucedido, qualidade de vida e saúde (ou bem-estar) surgem como conceitos, que tendo em comum o facto de partilharem uma conceção holista do ser humano se interpenetram, conjugam e condicionam dado que falar de envelhecimento bem sucedido implica necessariamente ter em linha de conta a longevidade e a saúde, mas também a satisfação, numa atitude de envolvimento ativo com a vida (Palmore, 2002). A qualidade de vida parece encontrar-se ligada ao conceito de envelhecimento bem-sucedido na medida em que este é eficaz quando os indivíduos conseguem manter os seus projetos de vida, as suas atividades de vida diária, bem como atividades físicas, cognitivas e sociais que lhes permitam ser autónomos (Paúl, 2005), percecionando, assim, a sua vida como uma vida com qualidade.

\section{EDUCAR PARA UM ENVELHECIMENTO BEM SUCEDIDO}

Nas leituras e reflexões que vamos fazendo acerca desta temática, constatamos que o envelhecimento da população foi, durante muito tempo, abordado numa perspetiva negativa relevando o amplo conjunto de problemas que dele derivam para a sociedade atual. Nos nossos dias, os ventos parecem de mudança dado que os documentos europeus mais recentes apresentam uma visão positiva do envelhecimento entendendo-o como uma oportunidade, valorizando os idosos e a contribuição que podem dar às comunidades em que se inserem orientando para 
a capacitação ou empoderamento dos indivíduos para um envelhecimento ativo e saudável (Comissão Europeia, 2011a, 2011b).

Do ponto de vista educacional, e no segmento das linhas orientadoras da Comissão Europeia, o envelhecimento da população nas sociedades desenvolvidas, mais do que um problema deve ser encarado como uma oportunidade de (trans)formação e, fundamentalmente, um indicador relevante da urgência de redefinição e ressignificação do sistema tradicional de educação, dado que a visão positiva do envelhecimento se alicerça na perspetiva da capacitação das populações e das comunidades para um envelhecimento bem sucedido, ou seja, num processo de educação/formação ao longo da vida.

Não obstante as várias teorias existentes para o explicar, as causas do envelhecimento são, ainda hoje, desconhecidas constituindo, por isso, um problema complexo. Parece, no entanto, apontar-se para o facto de «ser provável que o envelhecimento seja o efeito de uma quantidade de fatores (relacionados com o programa genético, o sistema imunitário, endócrino e nervoso ...) em interacção» (Simões, 2006, 33) e em relação com o meio, tendo o seu início no momento do nascimento.

Assim sendo o envelhecimento bem sucedido deve ser entendido como um processo que se prepara ao longo do ciclo vital, dado que as experiências naturais e culturais que vivenciamos parecem determinar significativamente a forma como envelhecemos.

Neste horizonte de visibilidade, o envelhecimento bem sucedido é uma questão de educação e educar para um envelhecimento bem sucedido é um processo que se inicia na infância, se prolonga na juventude e se intensifica na idade adulta. Um processo que proporciona os instrumentos e os conhecimentos necessários para que os indivíduos tomem as decisões mais acertadas face ao seu processo de desenvolvimento, dado a forma como se envelhece depender substancialmente da forma como se vive ao longo da vida. «O indivíduo é um agente do seu próprio processo de envelhecimento, e a capacidade para envelhecer bem -saudável e activo- depende de certa forma das decisões tomadas [...] e [do] repertório de comportamento aprendido ao longo da vida» (Fernández-Ballesteros, Robine, Walker e Kalache, 2013, 1).

Tendo em consideração esta forma de entender o envelhecimento bem sucedido deparámo-nos, por um lado, com a necessidade de repensar, em termos gerais e genéricos, as políticas educativas e suas finalidades na medida em que educar para um envelhecimento bem sucedido pressupõe políticas educativas que orientem para uma educação/formação facilitadora de recursos que promovam a autoformação participada de cidadãos informados, ativos e participantes do processo de construção e transformação de si mesmos e das comunidades em que se inserem.

Por outro lado, e no que concerne às realidades e necessidades educativas da população idosa, tendo em conta as recomendações da Unesco e do Conselho Europeu na aposta na educação ao longo da vida, novos desafios se colocam às 
políticas e finalidades educativas no sentido de garantir à população adulta e idosa a possibilidade de estar integrada num processo contínuo de educação-aprendizagem, tornando-se, para isso, urgente implementar novos contextos de educação e proporcionar os dispositivos adequados às necessidades, ritmos e disponibilidades deste novo público.

Muitos são os autores que entendem a educação como fator determinante de um envelhecimento bem sucedido (Simões, 2006; Ventosa, 2006; Dumazedier, 2001; Cuenca Cabeza, 2004; Osório, 2008; Pinto, 2008), sendo hoje consensualmente aceite que "a plasticidade cognitiva persiste apesar do avançar da idade» (Lemaire e Bherer, 2012, 430) continuando a ser possível aprender e desenvolver mecanismos favorecedores de uma melhor adaptação e/ou transformação das condições de vida.

Enquanto processo que procura criar condições de desenvolvimento de todas as capacidades e potencialidades do ser humano, a educação deve ser entendida como um processo de promoção de bem-estar e qualidade de vida, de desenvolvimento, satisfação e autorrealização pessoal. No caso vertente dos idosos, promover a qualidade de vida é, primordialmente, estimular à motivação para a ação, a aprendizagem, a participação e envolvimento nos vários contextos sociais, pois como temos vindo a referir o idoso que envelhece de uma forma ótima é aquele que permanece ativo e integrado familiar, social e culturalmente (Ribeiro e Paúl, 2011; Fonseca, 2005; Jacob, 2007).

Efetivamente, as dimensões axiais na avaliação da qualidade de vida da pessoa idosa são a saúde e os relacionamentos que favorecem a integração e participação sociocomunitária (Ramos, 2001). Deste modo as políticas públicas e intervenções educativas devem implementar medidas e programas que, promovendo a ligação entre tempos livres e educação/formação sensibilizem e promovam o acesso dos adultos e idosos a conhecimentos e atitudes que lhes permitam otimizar as condições de saúde física, o desenvolvimento psicológico e o fomento das relações interpessoais potenciando um envelhecimento ativo. Não enquanto conceito que pode significar uma «retórica vazia» (Boudiny, 2013) mas enquanto conceito que designa uma franja populacional que goza de bons níveis de autonomia, independência e qualidade de vida, não obstante a sua idade avançada.

Embora envelhecer não seja sinónimo de adoecer (Berger e Mailloux-Poirier, 1995), ou seja, embora o envelhecimento normal não seja uma doença, ocasiona modificações progressivas e irreversíveis que conduzem a um aumento na suscetibilidade de contrair doenças, na medida em que dado os seus mecanismos de defesa estarem mais debilitados, os idosos serem pessoas mais vulneráveis (Pérez, 2000).

Dado os grandes problemas que atingem a qualidade de vida dos idosos se focarem nas questões de saúde e sentimentos de perda de pertença e solidão, as áreas a trabalhar na educação dirigida à terceira idade devem ser a prevenção da doença e a promoção da saúde (Monteiro e Neto, 2008), assim como a dinamização 
de atividades promotoras do convívio e relacionamento interpessoal. Apesar da crescente vulnerabilidade e predisposição para a doença, com os avanços da ciência, nomeadamente da medicina, muitas das doenças crónicas com tendência a agravarem-se com o tempo, como as doenças de coração, hipertensão, cancro, diabetes, etc., podem ser prevenidas e/ou evitadas. Neste sentido, aprender e optar livremente por um estilo de vida saudável torna muito provável que o envelhecimento bem sucedido se vá tornando uma realidade cada vez mais ao nosso alcance, dado a doença e a incapacidade serem suscetíveis de prevenção (DGS, 2004).

Como referido, o estado de saúde da velhice vai-se delineando e construindo ao longo das fases etárias anteriores. Mais do que as consequências de um envelhecimento normal, a qualidade de vida e de satisfação dos idosos depende, em grande medida, do facto de terem optado, ou não, por um estilo de vida saudável durante as fases que precederam a velhice. Neste sentido, a prevenção e a educação/promoção da saúde surgem como um meio poderoso em defesa da sanidade física e mental da população idosa (Simões, 2006). A educação e promoção da saúde sendo um instrumento de natureza preventiva, com o objetivo de promover estilos de vida saudável e evitar comportamentos de risco potenciará, certamente, um relativamente elevado nível de saúde física, bem-estar psicológico e, consequentemente, melhor adaptação do indivíduo idoso, contribuindo significativamente para um envelhecimento bem sucedido.

Na perspetiva que temos vindo a defender e que pressupõe que a saúde não depende, fundamentalmente, dos profissionais de saúde e de atos médicos, mas sim dos comportamentos individuais e coletivos dos seres humanos, a educação para a saúde assume «uma importante função no âmbito da promoção da saúde e da prevenção da doença» (D'Espiney, 2004, 7), logo, constituir-se um fator decisivo na promoção de um envelhecimento bem sucedido. Sendo um instrumento de natureza preventiva, com o objetivo de promover estilos de vida saudável e evitar comportamentos de risco, a educação e promoção da saúde potenciará um relativamente elevado nível de saúde física, bem-estar psicológico e, consequentemente, melhor adaptação do indivíduo idoso.

Enquanto instrumento que possibilita a promoção deste estado de bem-estar assume todo o seu significado constituindo-se como uma tarefa a realizar ao longo da vida dos indivíduos. Parafraseando Simões $(2006,12)$ diremos que "não é, de facto, concebível uma política de prevenção sem a promoção da educação para a saúde» pois, esta constitui, incontornavelmente, a possibilidade da potenciação das mudanças de comportamentos e atitudes necessários ao estabelecimento de estilos de vida saudáveis. Ao dotar os indivíduos de capacidades que lhes permitam mudanças comportamentais positivas, no sentido de controlarem os fatores determinantes da saúde, a educação e promoção da saúde procurará educar no sentido de motivar para a construção de conhecimentos que conduzam à livre adoção de atitudes e comportamentos promotores de estilos de vida saudáveis constituindo-se, assim, num poderoso fator de intervenção de natureza preventiva. 
Estudos como os de Florindo (2008), Jesus (2010), entre outros, têm vindo a revelar que a educação tem implicações sobre o bem-estar e a qualidade de vida dos idosos. Alguns (Barros, 2014; Mártires, 2015) revelam que os principais benefícios da participação de idosos em dinâmicas educativas são as melhorias ao nível da saúde e o convívio, melhorando a perceção sobre a saúde e o bem-estar. O estudo de Cunha, Lopes e Pereira (2013) cuja finalidade é estabelecer uma relação entre a qualidade das redes sociais e a inclusão social, evidencia também que a educação tem um papel importante na aquisição de conhecimentos que favorecem a inclusão social e, consequentemente, os sentimentos de pertença e satisfação. Outros estudos desenvolvidos com pessoas idosas parecem indicar que a perceção de qualidade de vida e bem-estar está relacionada com o modo como as pessoas se sentem incluídas e vinculadas à comunidade em que se inserem, evidenciando efeitos positivos relativamente à perceção de bem-estar (Silva, 2012) e efeitos positivos/protetores contra a vulnerabilidade e fragilidade (Cramm e Nieboer, 2013).

A intervenção educativa revela-se uma dimensão fundamental no combate ao isolamento e solidão, possibilitando o alargamento do círculo de amigos, a integração e participação social, o enriquecimento cultural, a estimulação física e cognitiva, fatores que, como os estudos referidos comprovam, promovem um maior nível de bem-estar físico e psíquico que se traduz em sentimentos de confiança e autoestima, satisfação com a vida e felicidade pessoal.

Neste seguimento parece podermos dizer que a intervenção educativa com idosos, quer no âmbito da educação para a saúde, quer no âmbito das atividades artísticas, lúdicas e culturais promove as condições e conhecimentos favorecedores da manutenção das capacidades físicas e cognitivas e dos relacionamentos interpessoais potenciando a integração social e a capacidade de adaptação resultando em níveis significativamente mais elevados de bem-estar e desenvolvimento humano.

Assim sendo à educação coloca-se o desafio de integrar programas, projetos e ações educativas concebidos e implementados de forma a satisfazer as necessidades educativas deste novo público. Programas educativos que, partindo de uma conceção holista do fenómeno do envelhecimento, preparem adultos, jovens idosos e idosos para: a compreensão das mudanças características do envelhecimento; a vulnerabilidade e prevenção da doença; a mudança de papeis sociais; a manutenção das capacidades físicas e psíquicas e a sensibilização para atividades que favoreçam a redescoberta de novos sentidos para a vida (Fernández-Ballesteros, 2000; Fernandes, 2002), educando e formando para um envelhecimento bem sucedido. Neste mesmo sentido orientam também Os Planos Estratégico e Operacional da Parceria Europeia de Inovação para o Envelhecimento Ativo e Saudável (Comissão Europeia, 2011a, 2011b) ao apostarem fundamentalmente na Inovação, em formas diferenciadas e inovadoras de lidar com o envelhecimento, empoderando as pessoas e as comunidades através de programas educativos/formativos visando uma perspetiva proativa e emancipadora dos indivíduos capacitando-os para o autocuidado e para se manterem ativos e autónomos o mais tempo possível. 
Educar para um envelhecimento bem sucedido, ou uma gerontologia educativa (Osório, 2004) significa uma intervenção na e com a população adulta e idosa, quer de caráter preventivo, quer de caráter suplementar sobre o processo natural do envelhecimento promotora da aquisição de informações e conhecimentos que possibilitem às pessoas adultas e idosas planificarem estratégias para o seu envelhecimento, que ajudem a facilitar a adaptação pessoal e a autorrealização promovendo novos interesses e atividades que estimulem a vitalidade física e mental, tornando possível a qualidade de vida nesta última fase da vida.

Trata-se de uma intervenção educativa cuja finalidade é a promoção de uma vida ativa e autónoma mediante a aquisição de conhecimentos, saberes e atitudes facilitadores da continuidade da participação na vida individual e coletiva, da comunicação e das relações interpessoais. Educar para um envelhecimento bem sucedido significa promover o desenvolvimento intelectual ou empoderamento para a vivência de uma fase da vida entendida "como experiência acumulada, maturidade e liberdade para assumir novas ocupações» (Zimerman, 2000, 25).

A oferta educativa dirigida à terceira idade, no caso vertente de Portugal, tem sido escassa, no entanto mudanças significativas parecem marcar os nossos dias. Embora grandes desafios se coloquem, ainda, à educação no que concerne à população idosa constatamos uma crescente oferta de atividades e programas educativos para este segmento da população. A conjugação de diversos fatores tais como: o grande aumento da população idosa, a entrada precoce na reforma, a disponibilidade de tempo, a autonomia funcional e a longevidade, assim como a conscientização de que a educação é um instrumento precioso na preparação para esta fase da vida, tem impulsionado a emergência da oferta educativa, ainda que, no caso específico de Portugal, esta oferta apareça vinculada quase exclusivamente ao âmbito da educação não formal, sendo desenvolvida ao abrigo de instituições diversas da sociedade civil (Antunes, 2015).

A franja da população idosa que tende a ser cada vez maior, mais saudável e, também, mais instruída constituirá, certamente, num futuro próximo, um novo público alvo muito significativo da ação educativa. Assistiremos, incontornavelmente, a um aumento das habilitações literárias das novas gerações de idosos que (trans)formará a população idosa numa franja populacional "com mais probabilidades de ser civicamente mais consciente dos seus direitos e deveres, politicamente mais esclarecida, mais ativa e sanitariamente mais empenhada e evoluída» (Simões, 2006, 155), facto que aumentará significativamente a procura de educação/formação nesta fase tardia da vida.

As novas realidades e condições que se têm vindo a operar na franja da população idosa tornam premente a necessidade das políticas e práticas educativas se projetarem verdadeiramente, de direito e de facto, num processo de educação ao longo da vida colocando ao sistema educativo o desafio de se reinventar, reestruturar e flexibilizar no sentido de promover a sua implementação eficaz dentro e fora do sistema formal de educação. 
Assim, do ponto de vista educacional, entendemos que o envelhecimento da população, mais do que um problema, deve ser entendido como uma condição de progresso, de enriquecimento, de possibilidade de recontextualização e recriação de estruturas, temáticas, metodologias, públicos e educadores, no sentido da (re) estruturação de um sistema educativo que integre todas as fases da vida. Face às novas realidades, perceções e representações que vamos construindo da última fase da vida, sobretudo nas ditas sociedades desenvolvidas e em vias de desenvolvimento, esta redefinição, qualquer que seja a sua orientação deve, em nossa opinião, apostar de um modo muito particular, em instituições e programas/projetos educativos dirigidos aos adultos e idosos, implicando a participação e o apoio do Estado, pressupondo a colaboração entre o sistema público e os organismos da sociedade civil, configurando uma estrutura e uma rede mais abrangente, mais dinâmica e mais interativa capaz de promover um verdadeiro sistema de educação ao longo da vida de todos os indivíduos, orientado para a autoformação participada, empoderamento e emancipação.

\section{REFERÊNCIAS BIBLIOGRÁFICAS}

AARONSON, N. K. (1988) Quality of life: What is it? How should be measured? Oncology, 2, 69-74.

Alto Comissariado da SAúde (2011) Plano nacional de saúde 2011-2016. Lisboa, Ministério da Saúde.

ANTUNES, M. C. (2015) Educação e envelhecimento bem sucedido: a realidade portuguesa, en Pereira, J.; Lopes, M. e Rodrigues, T. (coords.) Animação sociocultural, gerontologia, educação intergeracional. Chaves, Intervenção, 109-116.

ATChley, R. C. (2000) Social forces and aging: an introduction to social gerontology. Belmont, Wadsworth.

Barros, D. (2014) Promoção do envelhecimento ativo: o contributo das práticas educativas de uma universidade da terceira idade. Dissertação de Mestrado. Instituto de Educação,Universidade do Minho.

Berger, L. e Mailloux-Poirier, D. (1995) Pessoas idosas. Uma abordagem global. Lisboa, Lusodidacta.

Boudiny, K. (2013) Active ageing: from empty rhetoric to effective policytool. Ageing and Society, 33 (6), 1077-1098. http://dx.doi.org/10.1017/S0144686X1200030X.

CANAVARro, M. (2010) Qualidade de vida e saúde: significados e níveis de análise, em CANAVARro, M. e SERrA, A. Qualidade de vida e saúde: uma abordagem na perspetiva da Organização Mundial de Saúde. Lisboa, Fundação Calouste Gulbenkian.

Carvalho, P. S. (2012) Etapas e processos de envelhecimento, em Moura, C. (org.) Processos e estratégias do envelhecimento: intervenção para o envelhecimento ativo. Porto, Euedito, 65-74.

Comissão EUROPEIA (2011a) Strategic implementation plan for the European innovation partnership on active and healthy ageing-strategic plan. Bruxelas, União Europeia. Consultado em 30 Agosto 2015. http://ec.europa.eu/research/innovation-union/pdf/ active-healthy-ageing/steering group/implementation-_plan.pdf. 
COMISSÃO EuROPEIA (2011b) Strategic implementation plan for the European innovation partnership on active and healthy ageing-operational plan. Bruxelas, União Europeia. Consultado em 30 Agosto 2015. http://ec.europa.eu/research/innovation-union/pdf/ active-healthy-ageing/steering-group/operational_plan.pdf.

Cramm, M. e Nieboer, P. (2013) Relationships between frailty, neighborhood security, social cohesion and sense of belonging among community-dwelling older people. Geriatrics \& Gerontology International, 13 (3), 759-763. http://dx.doi.org/10.1111/j.14470594.2012.00967.x.

Cuenca Cabeza, M. (2004) Ócio e animação sociocultural: presente e futuro, em Trilla, J. (coord.) Animação sociocultural: teorias, programas e âmbitos. Lisboa, Instituto Piaget, 335-349.

Cunha, P.; Lopes, M. e Pereira, A. (2013) Papel da educação para a promoção do suporte social e nível de satisfação com a vida, em Congresso internacional de pedagogia social. Oviedo, Universidade de Oviedo, 228-235.

D'Espinay, L. (coord.) (2004) Implementação do processo de Bolonha a nível nacional-grupos por área de conhecimento: enfermagem. Lisboa, Ministério da Ciência, Tecnologia e Ensino Superior.

Direç̧ão Geral SAÚde (2004) Plano nacional para a saúde das pessoas idosas. Lisboa, Dgs.

Donald, K. (1997) Coming of age: the virtual older adult learner. Continuing education conference. June 1-4, Canadá.

Dumazedier, J. (2001) Lazer e cultura popular. S. Paulo, Perspectiva.

Esteves, A. J. (1995) Jovens e idosos. Família, escola e trabalho. Porto, Edições Afrontamento.

FERNANDES, E. (2002) Cérebro racional e cérebro sócio-emocional nas aprendizagens, nos comportamentos e na saúde. Vagos, Edioanta.

Fernandes, P. (2000) A depressão no idoso. Coimbra, Quarteto.

Fernández-BAllesteros, R. (coord.) (2000) Gerontología social. Madrid, Ediciones Pirámide.

Fernández-Ballesteros, R.; Robine, J. M.; Walker, A. e Kalache, A. (2013) Active aging: a global goal. Current Gerontology and Geriatrics Research, Volume 2013, 1-4. http://dx.doi.org/10.1155/2013/298012.

FloRINDO, G. (2008) Transição para a reforma no concelho de Évora: assimetrias sócio-educativas entre urbanidade e ruralidade. Dissertação de Mestrado. Faculdade de Ciências Sociais e Humanas, Universidade Nova de Lisboa.

FonseCA, A. M. (2005) Desenvolvimento humano e envelhecimento. Lisboa, Climepsi Editores.

Fontaine, R. (2000) Psicologia do envelhecimento. Lisboa, Climepsi Editores.

GeIs, P. (2003) Atividade física e saúde na terceira idade: teoria e prática. Porto Alegre, Artmed.

JACOB, L. (2007) Animação de idosos: actividades. Porto, Âmbar.

Jenny, M. E. (1997) Measuring quality of life. Archives of disease in childhood, 77, 347-354. http://dx.doi.org/10.1136/adc.77.4.347.

JEsus, A. (2010) A importância da universidade sénior na qualidade de vida e solidão dos seniores de Gondomar. Dissertação de Mestrado. Instituto Superior de Serviço Social do Porto.

Kaplan, R.; Saluis, J. F. e Patterson, T. L. (1998) Health, and human behavior. New York, McGraw-Hill, Inc.

Lemair, P. e Bherer, L. (2012) Psicologia do envelhecimento. Uma perspetiva cognitiva. Lisboa, Instituto Piaget. 
Levi, R. e Drotar, D. (1998) Critical issues and needs in health-related quality of life assessment of children and adolescents with chronic health conditions, em DrOTAR, D. (ed.) Measuring health-related quality o life in children and adolescents-Implication for research and practice. New Jersey, Lawrence Erlbaum Associates Publishers, 92-127.

LORDA, C. R. (2001) Recreação na terceira idade. Rio de Janeiro, Sprint.

martínez García, A.; Carrera Sáez, J. e Haro escarbajal, A. (2000) Educación para la salud. La apuesta por la calidad de vida. Madrid, Arán Ediciones.

MÁrtires, M. A. (2015) Envelhecimento ativo e autonomia: idosos com doença crónica, em Pereira, J.; Lopes, M. e Rodrigues, T. (coords.) Animação sociocultural, gerontologia, educação intergeracional. Chaves, Intervenção, 37-45.

Monteiro, H. e Neto, F. (2008) Universidades da Terceira Idade da solidão aos motivos para a sua frequência. Oliveira de Azeméis, Livpsic.

Murga, Á. (2004) Salud y desarrollo humano sostenible: las competências de la educación, em Quesada, R. P. (org.) Educación para la Salud. Madrid, Ediciones Díaz de Santos, 81-99.

Osório, A. (2004) Animação sociocultural na terceira idade, em Trilla, J. (coord.) Animação sociocultural: teorias, programas e âmbitos. Lisboa, Instituto Piaget, 251-263.

Osório, A. (2008) Animação sociocultural na terceira idade, em Pereira, J.; VieItes, M. e LOPES, M. (coords.) A animação sociocultural e os desafios do século XXI. Chaves, Intervenção, 207-219.

Osório, A. e Cabral, F. (coords.) (2007) As pessoas idosas. Contexto social e intervenção educativa. Lisboa, Instituto Piaget.

OMS (1948) Carta fundamental da Organização Mundial de Saúde.

OMs (1978) Declaração de Alma-Ata. Primeira conferência internacional sobre cuidados primários de saúde. Alma-Ata, uRss.

OMs (1986) Carta de Ottawa. Primeira conferência internacional para a promoção da saúde. Ottawa, Canadá.

oms (2001) Men, ageing and health. Achieving health across the span. Geneve, oms.

OMs (2002) Active ageing: A policy framework. Geneve, oms.

Palmore, E. B. (2002) Successful aging, em Encyclopedia of aging.

Paúl, M. C. (1996) Psicologia dos idosos: o envelhecimento em meios urbanos. Braga, S.H.O.

Paúl, M. C. (2005) Envelhecimento ativo e redes de suporte social. Porto, ICBAS-UP.

PAIs Ribeiro, J. (2005) Introdução à psicologia da saúde. Lisboa, Quarteto Editora.

PARENTE, M. A. (2006) Envelhecimento: um processo adaptativo multifatorial, em PARENTE, M. A. (org.) Cognição e Envelhecimento. Porto Alegre, Artmed, 17-18.

Pérez, M. A. (2000) Guía de cuidados de personas mayores. Madrid, Síntesis.

PINTO, M. (2008) Da aprendizagem ao longo da vida ou do exemplo de uma relação ternária: agora, antes, depois. Porto, Cadernos de Apoio Pedagógico da FluP.

Pontes, J. L. (2012) Promoção da qualidade de vida, em Moura, C. (org.) Processos e estratégias do envelhecimento. Porto, Euedito, 289-296.

Portella, M. (2004) Grupos de terceira idade: a construção da utopia do envelhecer saudável. Passo Fundo, UPF.

RAfart, J. V. (2001) Epidemiología general de las enfermedades crónicas. Estratégias de prevención, em GiL, P. (org.) Medicina preventiva y salud pública. Barcelona, Masson, 647-662.

Ramos, H. V. (2001) Qualidade de vida e envelhecimento, em ArCHER, L.; Biscaia, W. O. e Renaud, M. (coords.) Novos dasafios à bioética. Porto, Porto Editora, 225-231. 
Ribeiro, J. L. P. (1994) Reconstrução de uma escala de locus de controlo de saúde. Psiquiatria Clínica, 15 (4), 207-214.

Ribeiro, J. L.; Meneses, R. F.; Meneses, I. e Grupo-Qvd (1998) Avaliação da qualidade de vida em crianças com diabetes tipo 1. Análise Psicológica, XVI (1), 91-100.

Ribeiro, O. e Paúl, C. (coords.) (2011) Manual do envelhecimento activo. Lisboa, LidelEdições Técnicas.

Silva, M. (2012) Sentido de comunidade e bem-estar em idosos. Dissertação de Mestrado. Faculdade de Psicologia e Ciências da Educação, Universidade do Porto.

Simões, A. (2006) A nova velhice. Um novo público a educar. Porto, Ambar.

SPIETH, L. E. e Harris, C. V. (1996) Assessment of health-related quality of life in children and adolescents: An integrative review. Journal of Pediatric Psychology, 21, 175-193. http://dx.doi.org/10.1093/jpepsy/21.2.175.

Ventosa, P. V. (2006) Perspectivas actuales de la animación sociocultural: cultura tiempo libre y participación cultural. Madrid, Editorial ccs.

Wong, P. (1989) Personal meaning and successful aging. Canadian Psychology, 3-30, 516525. http://dx.doi.org/10.1037/h0079829.

Zimmerman, I. G. (2000) Velhice. Aspectos biopsicossociais. Porto Alegre, Artemed. 